\title{
EXPERIMENTAL EVALUATION OF CIRCUIT BOARD COMPONENTS UNDER EXTREME CONDITIONS
}

\author{
Krzysztof SOKÓk ${ }^{*}$, Piotr PTAK $^{*}$ \\ *Institute of Mechanic and Machine Design Foundations, Czestochowa University of Technology, \\ ul. J.H. Dabrowskiego 69, 42-201 Częstochowa, Poland \\ krzysztof.sokol@pcz.pl, piotr.ptak@pcz.pl
}

received 12 May 2021, revised 10 October 2021, accepted 12 October 2021

\begin{abstract}
Designing products operating in harsh conditions is a challenging task. Years of experience, developed standards and good practices are crucial in achieving the intended result. The article shows a methodology for designing electronic systems based on the worst-case analysis (WCA) and comparing its outcomes with the experimental verification of an actual circuit through large-scale tests. The analysed diode-based semiconductor circuit is part of a temperature measuring system of industrial application. The objective of the design and analysis process is to achieve a reliable solution, which has all the required functionalities under actual, extreme operating conditions. The preliminary circuit design is developed using ideal components. The truth table, which represents customer requirements, is created to check the correct operation of the system. Simulation software, such as LTSpice, are used as the main tools to verify the correct functioning based on ideal or close-to-real component models. Next, based on the results of computer simulations, the WCA is conducted, considering all extreme (worst) operating environment parameters, such as, among others, ambient temperature or ageing. WCA results were verified through an experimental, large-scale measurement of the real system, with defined forward voltage as a function of the current flowing through the semiconductor at various ambient temperatures.
\end{abstract}

Key words: experimental test, mathematical model, semiconductor diodes, thermal chamber, WCA

\section{INTRODUCTION}

Worst-case analysis (WCA) is a popular tool for verifying a correctly designed system. This analysis is an effective tool to check a design, to ensure, with high probability that potential damage and imperfections are identified and eliminated before manufacturing and delivery to the customer. It is a qualitative assessment of a product's performance, taking into account the impact of external factors, such as the operating environment or component ageing. Besides analysing circuits, WCA often involves thermal stress and searching for extreme nominal value tolerances, failure variants or reliability prediction. The primary objective of the WCA is checking design correctness aimed at ensuring operation in accordance with the complete specification, throughout its service life and in the worst conditions and tolerances, such as ageing, initial deviations or temperature. Stress analysis is aimed at improving reliability by ensuring a sufficient margin, relative to permissible stress limits set out in the component documentation. A correctly conducted analysis mitigates overload conditions, which can contribute to a failure or reduce the change rate of parameters induced by thermal stress throughout the product's life cycle. The WCA should be conducted for all circuits covered by the design, regardless of their criticality. The WCA is an analytical technique that involves the observation of defined environmental conditions, such as external stresses. Operating conditions include electrical inputs/outputs, component quality, correlations between the parts and the impact of time on subassembly parameters. WCA is a key tool in the process of developing a multi-system platform, which forms a complete product only as a whole. [1] discussed WCA methodologies based on National Aeronautics and Space Administration (NASA) experience, to develop a database containing worst variants for parts, sensitivity analysis and a mathematical approach to the extreme value analysis (EVA), root sum square (RSS) and the Monte Carlo analysis for solving circuit equations. These analyses have become an accepted industry standard over the last 8 years.

Scientists [2-9] showed the results of tests involving such methods as reverse engineering, which is required to find regions and prepare for analysing them. They also state worst-case execution time (WCET) analysis and discuss their accuracy. The authors also presented qualitative and quantitative data regarding the program structure of the regions. This information is suitable in terms of evaluating whether WCET analysis can provide useful results for this class of real codes, without excessive manual labour. A list of WCET analytical tool functions, which appeared during the project, was also presented.

Ferlet-Cavrois [10] described the worst deviation case for silicon on insulator (SOI) transistors in two technologies. It also includes experiments and simulations for the threshold shift analysis of an SOI transistor voltage. Lynch [11] analysed the worst output voltage ratio for resistor memory matrix having a finite resistance coefficient for bit elements. It was concluded that the resistance coefficient does not have to be high, and ratios $>10$ are usually sufficient. It also discusses trade-offs in terms of output 
power and output voltage. Marcovitz [12] analysed and developed procedures for designing a transistor system coupled with a resistor and used in the mechanization of logic operations. The basis circuit consists of one transistor and several resistors. The circuit performs the OR function, followed by NO or AND, and then NO. Owing to the mechanization of these complex functions, it is possible to create any logical system. The first operational prerequisite for this circuit is that a transistor must be saturated if one or more inputs are of low status. The second requirement is transistor isolation if all inputs are high. A WCA is conducted for each of these requirements. Three solution types, namely, general purpose, indirect (flexible) purpose and special purpose were reviewed. Tian [13] demonstrated that the operations of an analytical and precise algorithm are complementary - the analytical algorithm is faster than the precise algorithm but has a higher interval spread error. It also discusses AC tolerance analysis, time-domain tolerance analysis and DC tolerance analysis techniques. Several examples and comparisons are given. In [14] the technique for characterising the behaviour of a DC-powered converter within the common mode (CM) was introduced for the first time. This characterization technique is based solely on measurements conducted on power converter terminals, without the requirement of in-depth knowledge regarding the converter structure, and describes CM behaviour in relation to the Thevenin equivalent circuit. Next, the analysis is used to determine the upper limit of CM potential within a system with at least one power converter. Finally, a model of the Thevenin equivalent circuit was generalised to a dual-port equivalent circuit, owing to which $\mathrm{CM}$ behaviour can be modelled at both the input and output of a power converter. Spence [15] provided an overview of concepts involving tolerance analyses and electronic system designing. They thoroughly discussed the approach towards statistical analyses. Such statistical methods as the Monte Carlo analysis were presented. Finally, they discussed several application examples for such analyses in terms of designs. Divekar [16] discussed statistical analyses simulating circuit performance variations caused by tolerance variations or other factors related to circuit manufacturing. The statistical circuit simulation procedure was illustrated via a simple system undergoing measurements. The measurement data are compared with the simulation results from worst-case and statistical analyses, without correlating model parameters and with model parameter correlations for devices used within the circuit. This comparison indicates a need to correctly take model parameter correlations into account. Riley [17] and Sokół et al. [18] focussed on the WCA in terms of using resistors in electronic systems. Hillebrand [19] reviewed the techniques and tools used to verify Verisoft software. Nassif [20] showed an approach towards WCA, which leads to more realistic estimates of the electronic device and circuit performance changes. Rafaila et al. [21] discussed experiment design concepts, and screening tests aimed at reducing the verification space size, whereas Maly et al. [22] and Nassif et al. [23] suggested a methodology for modelling random process fluctuations, together with its advantages and applications.

This research paper includes the results of studies focused on an electronic system that can be used, among others, as a temperature sensor or resistor calibrator. WCA and experimental measurements were used to determine the forward voltage as a function of the current flowing through the semiconductor at various ambient temperatures.

\section{ANALYZED SYSTEM}

The electronic system analysed by the authors is a module responsible for providing information on the engine operating temperature. The mathematical model of the analysed circuit is shown in Fig. 1 and described by Eq. (1). Diodes within the system act as a temperature sensor consisting of an actuator made up of two serially connected semiconductor diodes, contained in a single enclosure. The authors of the design decided to use two light-emitting diodes LEDs owing to the increased measurement accuracy. Two LEDs connected in series supply higher voltage to the output, which at the same time is the average value of two voltages. The application of two semi-conductors contained in a common enclosure enables ensuring the same operating conditions, smaller dimensions and minimizing the costs associated with the requirement to use subsequent LEDs in separate enclosures. A resistor limiting current consumption and a capacitor filtering the output voltage were also used within the analysed system. Semiconductor diodes are very popular, relatively inexpensive and accurate temperature measuring systems. A linear temperature coefficient, such as $-2 \mathrm{mV} /{ }^{\circ} \mathrm{C}$ for the operating temperature, makes LEDs an excellent solution for flexible and lowcost applications. A diode-based system can be simple, but its implementation may require a deeper analysis.

$I=I_{S}-I_{D 1} \exp \left(\frac{V-I_{S} R_{01}}{A_{01} V_{T}}\right)-I_{D 2} \exp \left(\frac{V-I_{S} R_{01}}{A_{02} V_{T}}\right)-I_{C}$

where: I - output current, Is - current supplied by a generator, $I_{D 1}$ - diode 1 current, ID2 - diode 2 current, IC - capacitor current, $A_{01}, A_{02}$ - ideality factor corresponding to diodes 1 and 2, respectively, $V_{T}$ - thermal voltage, $V$ output voltage, resistance $R_{01}$ and $R_{02}$.

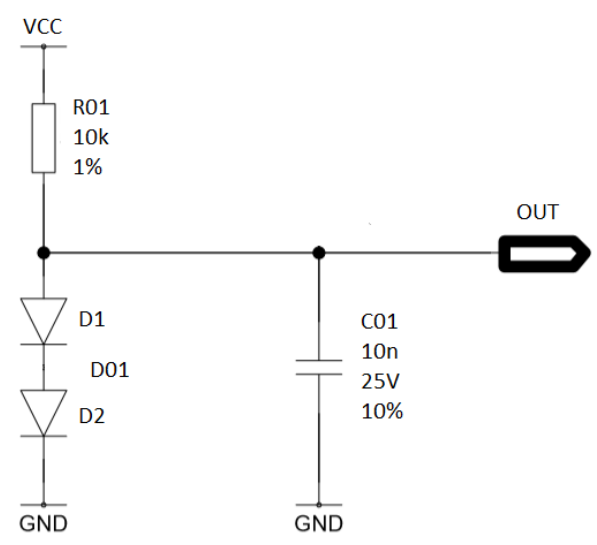

Fig. 1. Circuit diagram

Authors [24 - 26] have identified typical temperature measurement techniques and focused on the applications and advantages of silicon diodes acting as temperature sensors in various operating conditions. This article verified the system shown in Fig. 1 in terms of its use as a resistance calibrator in an unchanged version. R01 resistor in the analysed system is replaceable, which enables resistance measurements of this component (in this system, the resistor acts as a current limiter, hence inducing a determined voltage drop on the diodes). Voltage is read directly by the microcontroller's ADC input. The output signal is filtered by the capacitor. 


\section{OPERATING CONDITIONS AND SELECTION OF COMPONENTS}

The analysed system should perform all its functions in real operating conditions that can be encountered during the normal operation of the system. Requirements for the analysed module are as follows:

- Operating voltage VCC $(5 \mathrm{~V} \pm 0.25 \mathrm{~V})$

- Operating temperature $\left(-40^{\circ} \mathrm{C} ; 150^{\circ} \mathrm{C}\right)$

- Flowing current (D01) $(100 \mu \mathrm{A} ; 1500 \mu \mathrm{A})$

Initial simulation of the system, conducted using the LTSpice tool, enabled proceeding to the stage of selecting suppliers and specific component models. The following system components were selected:

- Capacitor - Kemet C0603C103F3GACTU

and the following LEDs were selected for further analysis:

- Diode - ROHM BAV99HMFH (SOT23)

- Diode - NXP BAV99W (SOT323)

- Diode - Onemi BAV99W (SOT323)

\section{WORST-CASE ANALYSIS}

The worst-case circuit analysis is an analysis technique, which determines circuit performance in the worst case scenario (extreme environmental and operating conditions). A series of calculations verifying the correct system functioning were conducted; however, this paper is limited to discussing temperature measurement.

For this case, it was assumed that the resistance of the $R_{01}$ resistor is constant over the entire temperature range, which enabled focusing the calculations solely on the $I_{F}\left(V_{F}\right)$ forward current characteristics depending on the forward voltage, which was studied for the entire temperature range and a certain range of expected current flowing through a diode series.

The $I_{F}\left(V_{F}\right)$ forward current characteristics depending on the forward voltage of selected diodes are shown below. Analysing and comparing the technical documentation of electronic components from different manufacturers entail numerous difficulties for the designer. The greatest hindrance when comparing the parameters is the different conditions of the tests providing the data shown in the technical documentation on manufacturers' websites. Each manufacturer individually defines the test conditions for component verification, which provide the data in the form of a table or specification (Figs. 2-4).

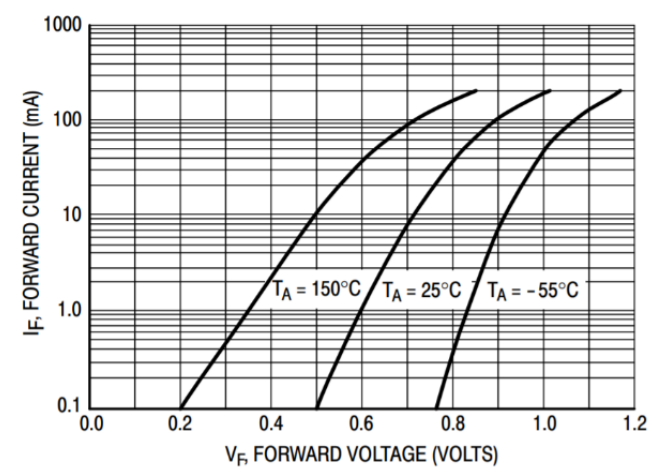

Fig. 2. Onsemi BAVg9W $I_{F}\left(V_{F}\right)$ datasheet (https://www.onsemi.com/pdf/datasheet/bav99wt1-d.pdf)

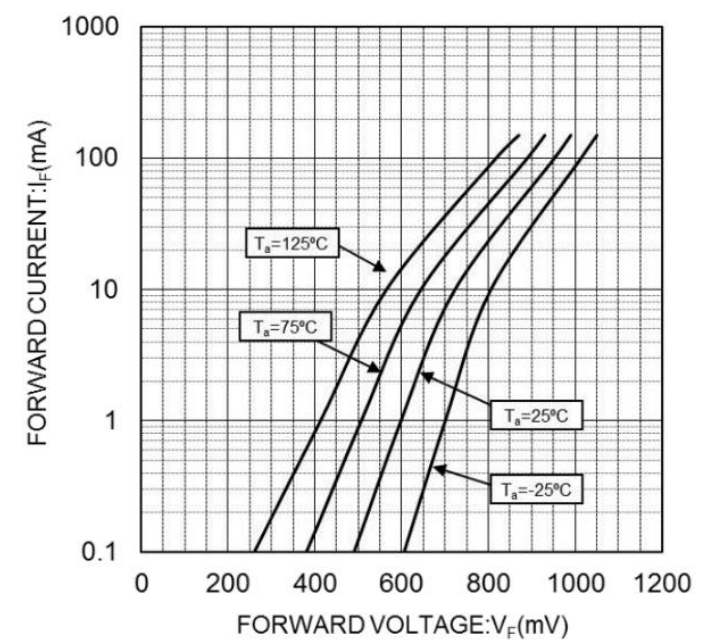

Fig. 3. ROHM BAV99HMFH $I_{F}\left(V_{F}\right)$ datasheet (https://pl.mouser.com/ datasheet/2/348/bav99hmfht116-e-1801281.pdf)

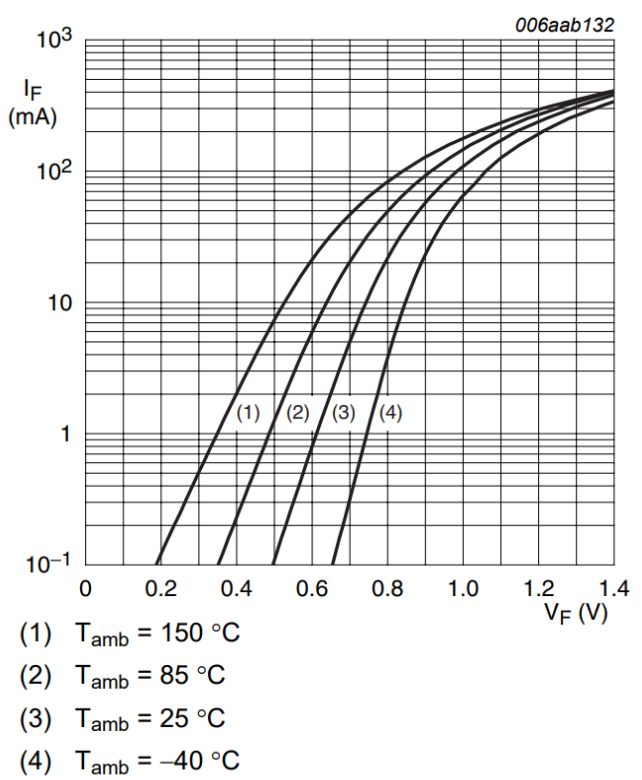

Fig. 4. NXP BAV99W $I_{F}\left(V_{F}\right)$ datasheet (https://www.elfadistrelec.pl/ Web/Downloads/_t/ds/bav99-series_eng_tds.pdf)

The technical documents made available by the component manufacturers very often include incomplete information regarding the conditions, in which the charts or tabular data were obtained. Very often, due to the specifics of a project, it is quite difficult to fully adopt the technical documentation data in a specific design. In many cases, this leads to a big problem in terms of how to supplement the calculations with component parameters, which do not fully correspond to the analysed situation in the designed system. Another difficulty is the manner of how the component vendors present the component parameters. The following types can be distinguished in this regard:

- Presentation of complete characteristics for a full range of temperatures and currents (Figs. 2-4) but without a statement whether they are nominal, minimum or maximum values. This forces an engineer to make assumptions and adopt a high safety factor for the calculations.

- Presentation of characteristics for one or two temperatures only, which significantly hinders the analysis involving the be- 
haviour of the component in negative temperatures. As a result, based on two functions, one has to assume the hypothetical behaviour at a given temperature.

- Presentation of parameters for one ambient condition only, as a constant operating within the stated range.

- No information about a particular operating parameter of the component is given, despite the fact that such a parameter may be crucial in certain applications.

In the case of components such as resistors, capacitors or diodes, the operating conditions in the case of static characteristics are mainly limited to voltages, currents and ambient temperature, whereas in the case of simulating complex integrated circuits or dynamic analysis, the list of operating conditions during a simulation grows significantly, entailing issue in respect of a reliable functional verification of the system.

\section{EXPERIMENTAL MEASUREMENTS AND TEST SETUP}

Electronic systems often experience problems with the determination of certain boundary conditions, such as voltage and current levels or the ambient temperature and self-heating of individual components on printed circuit board (PCB). An approximate estimation of hard-to-determine boundary conditions, aimed at solving the issue via a direct method, may lead to significant errors. This paper presents an inverse method, which enables determining a transient temperature field as accurately as possible. This method is based on analysing a large-scale test in specific, repeatable environmental conditions. It can be applied when solving simple unidimensional problems, as well as simple and complex multi-dimensional problems.

The article presents the results of experimental tests involving a semiconductor diode from three different manufacturers. The conducted laboratory tests standardised the diode test conditions, which enabled a reliable comparison of their characteristics and their numerical analysis. The outcome of these analyses was a conclusion regarding the manufacturer ensuring the highest accuracy and linearity of the analysed characteristic.

LED series from three manufacturers were ordered for the laboratory tests. For the verification of the actual manufacturing spread to be as clear as possible, 136 pieces (128 used, 8 spares) from different stores were ordered, which allowed to potentially receive diodes from different manufacturing batches.

A dedicated PCB was designed for the study (Figs. 5 and 6), which enables correct and effective implementation of the laboratory tests.

Designed PCB is the result of a previously established measurement methodology (Fig. 7). It involves the possibly most accurate determination of voltage on diode pairs with a correctly designed PCB, which enables omitting the voltage drop on the channels and conduits directly connecting diode pairs with a scopecorder. For this purpose, the lengths of the used wires were the same, and the channels on the laminate were routed so that their lengths were the same.

Measurements of the forward voltage drop during the lifetime of three diode models were conducted on dedicated PCBs (Figs. 5 and 6). Every supplier had 128 enclosures. Each enclosure houses two LEDs, where the double voltage drop for both diodes was measured. All semiconductors were placed on two PCBs with an independent power supply.

Voltage was measured for the complete temperature range (measurement conducted for 9 different temperatures) and 15 current levels. LED voltage was measured during the tests using a scopecorder. It was decided to use this measuring device because of its modularity and multi-channel nature. This measuring device can be configured for multiple applications. It combines the advantages of a rapid oscilloscope and a traditional data recorder in a single portable instrument. Owing to the possibility of a scopecorder measuring 16 diodes simultaneously, the measurement was repeated until all LEDs were measured (for each temperature).

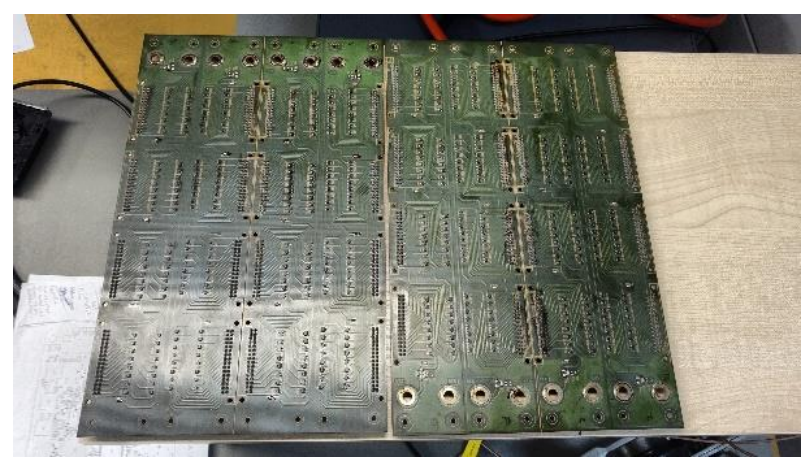

Fig. 5. PCB prepared for the development test. PCB, printed circuit board

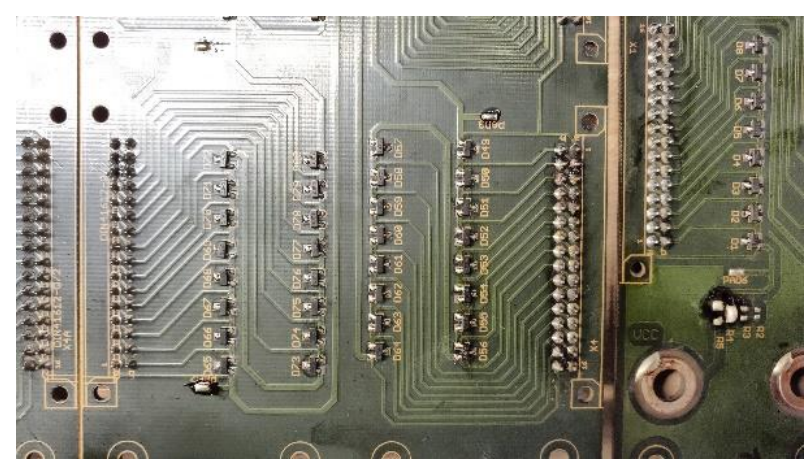

Fig. 6. PCB prepared for the development test (detailed view). PCB, printed circuit board

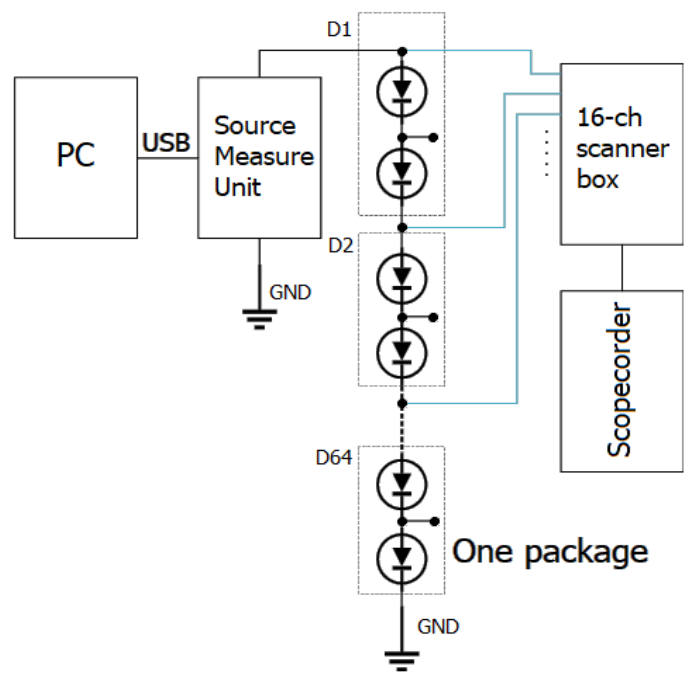

Fig. 7. Circuit diagram

The dedicated PCB was embedded in a specialized thermal chamber used to conduct all controlled climatic tests for a random period of time and on-going monitoring of interior conditions 
(Fig. 8). Such climatic chambers are commonly used for testing the stability of medicinal products in the pharmaceutical industry but also in the construction industry, where, among others, it conditions concrete samples. It is also used in the automotive industry and the widely understood electronic engineering, for environmental testing of sub-assemblies and devices.

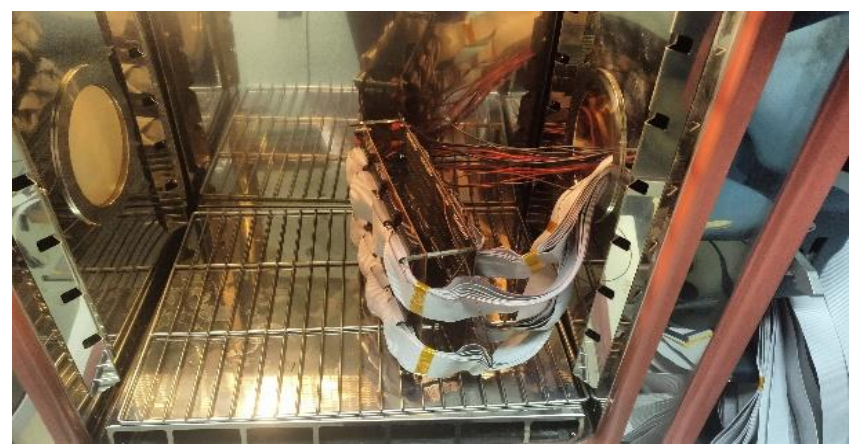

Fig. 8. PCBs in a thermal chamber. PCB, printed circuit board

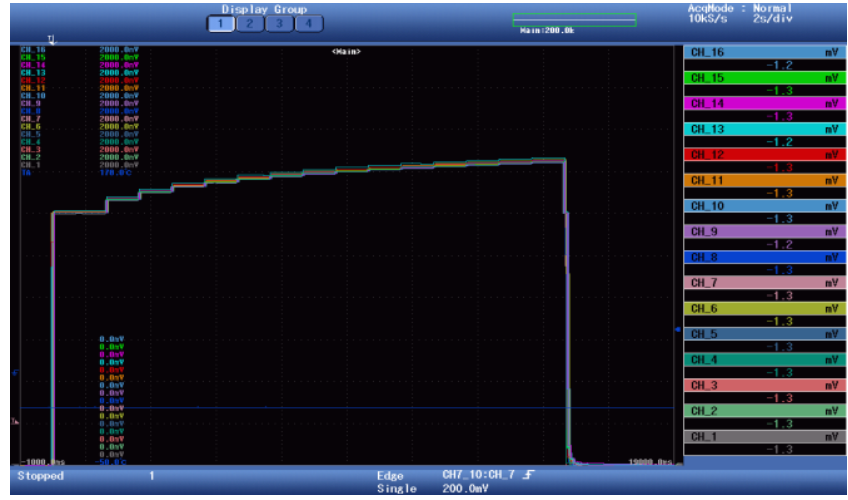

Fig. 9. Post-test scopecorder screen

Every diode pair was connected via a tape to a scopecorder recording the voltage as a function of time. A DC load power supply, controlled through a computer app, was connected to each of the printed boards. A procedure involving a computer setting a current excitation in the range of $100-1,500 \mu \mathrm{A}$, with an increment of $100 \mu \mathrm{A}$ and increment duration of $1 \mathrm{~s}$ was conducted for each of the analysed temperatures. An example of obtained time waveforms from a scopecorder screen is shown in Fig. 9. They show voltage levels for a series of diodes, depending on the induced time-variable current flowing through them.

\section{RESULTS}

A series of data for analysis were obtained based on the conducted laboratory testing. The compiled data were analysed in terms of finding the optimal component for the project. For this purpose, the authors conducted a series of calculations enabling a comparison of all three LEDs. The first step involved calculating the average values for each of the tested diodes, in each of the tested currents, for a full range of temperatures Eq. (2).

$n=\left(\sum_{i=1}^{k} n_{i}\right) / k$ where: $k$ - number of tested samples, $n$ - arithmetic mean; and $n_{\mathrm{i}}$ - ith value in a dataset.

Next, the standard deviation was calculated according to Eq. (3), along with the previously calculated arithmetic mean Eq. (2). Efron [27] and Zięba [28] wrote about fitting methods, which involve algorithms for fitting various functions, a study of fitting quality and describe new statistical methods, such as the robust statistic, analysis of self-correlated data and Monte Carlo modelling applications.

$\sigma=\sqrt{\frac{\sum_{i=1}^{k}\left(n_{i}-n\right)^{2}}{k-1}}$

where: $\sigma$-standard deviation.

The next step involved calculating the six sigma coefficient Eq. (4). The six sigma value was determined empirically. It was noticed that when processes are composed of hundreds of steps with the possibility of making an error in each of them, the probability that the entire process creates a good product at the first attempt depends on the product of probabilities of correct execution of each step. It was empirically established that even in the case of very complex processes, assuming six sigma at every stage, there was a high chance of obtaining a good product without corrections. This methodology was promoted by Harry et al. [29]. He derived them based on observation and practice. They are currently used as a change model since the production defects are not subject to the normal distribution in many cases. Standard deviation is not used to calculate potential defects in such a case, but the parameters of variabilities typical of other types of statistical distributions, which are discussed in greater detail in Yang et al. [30] and White [31].

$\pm s=n \pm 6 \sigma$

The measured and calculated parameters are shown in Figs. 10-12 and compared with manufacturers' datasheet. The next step involved selecting the range of expected current flowing through a diode to determine the graph linearity for a given range. Eq. (5) was used for this purpose. It enables determining a surface area formed by the mean (ideal) function of the voltage increase value $f(x)$ and the real value of this increase $g(x)$. The geometric interpretation of Eq. (5) is shown in Fig. 13.

$|D|=\int_{a}^{b}[f(x)-g(x)] d x$

where: $x$ - test point, $a$ - starting test point $(100 \mu \mathrm{A}), b$ - end test point $(1,500 \mu \mathrm{A}), f(x)$ - ideal function and $g(x)$ - real-valued function.

The outcomes of the conducted calculations following formulas (2)-(5) are the results presented below. Figs. 10-12 contain the forward current as a voltage current function $-I_{F}\left(V_{F}\right)$ for each manufacturer. The blue colour in the graphs marks the mean value obtained from a series of measurements. The grey and yellow colours mark the plus and minus six sigma values, respectively. Orange colour curves correspond to the theoretical values from technical documentation. When analysing these graphs, it can be seen that the average measured, as well as theoretical datasheet values differ the most for Onsemi diodes under higher currents (Fig. 10). 


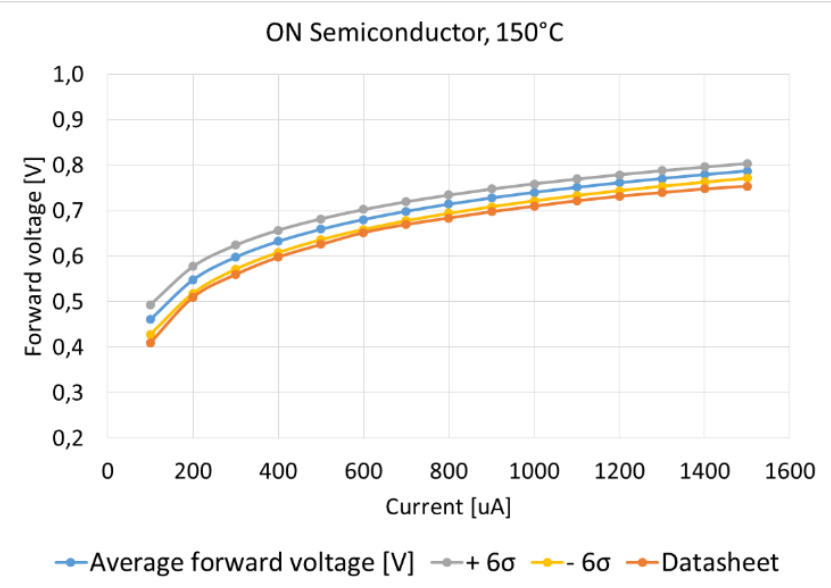

Fig. 10. Test results - Onsemi $150^{\circ} \mathrm{C}$

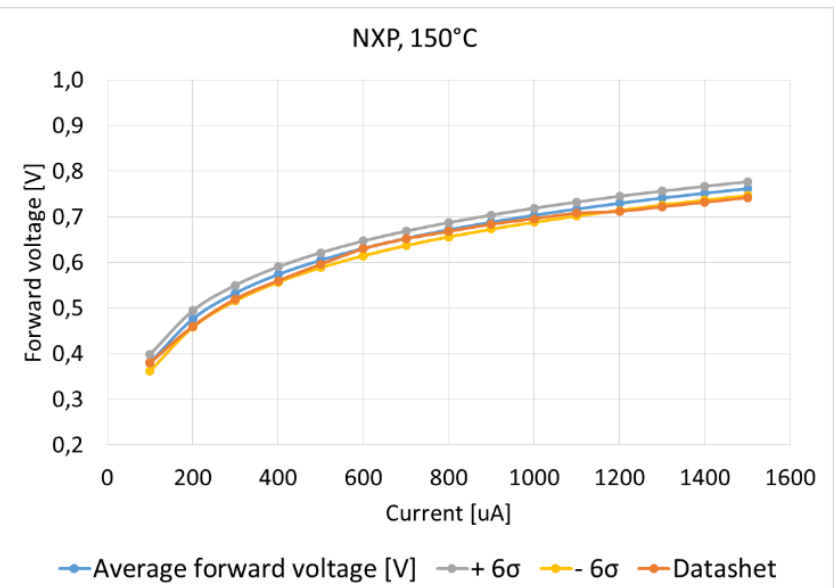

Fig. 11. Test results $-\mathrm{NXP} 150^{\circ} \mathrm{C}$

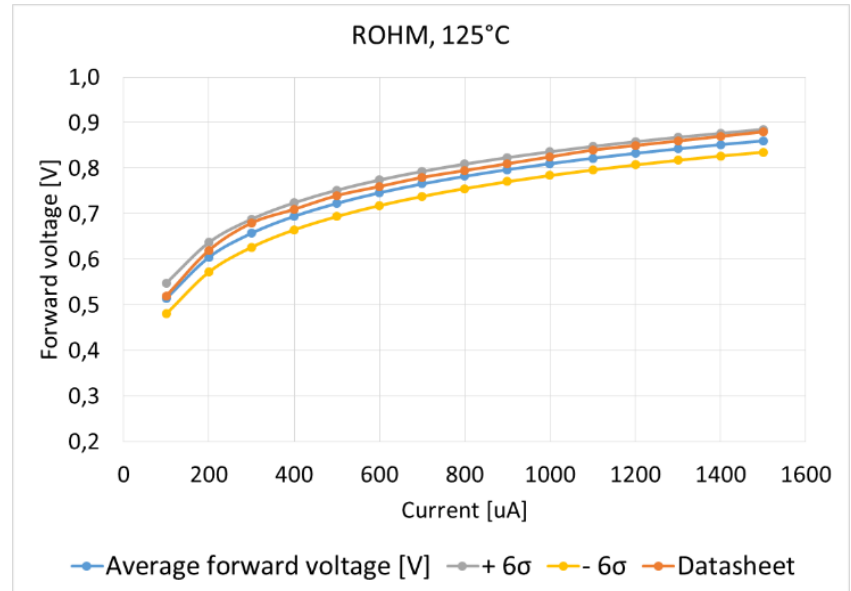

Fig. 12. Test results $-\mathrm{ROHM} 125^{\circ} \mathrm{C}$

The lowest average differences between the test and documentation can be observed for NXP LEDs, and amount to $13 \mathrm{mV}$, with $16 \mathrm{mV}$ for ROHM and $42 \mathrm{mV}$ for Onsemi. The observed differences can have several reasons. First, it should be noted that the manufacturer does not usually provide a formula for a function describing the curve. Second, please note that the $I_{F}\left(V_{F}\right)$ function for each of the analysed diodes is not fully linear, which is confirmed both by manufacturer data, as well as laboratory measurements. On the other hand, each of the semiconductors is characterized by the linearity of the current $>400 \mu \mathrm{A}$. This statement is true for all three manufacturers at each of the tested temperatures. Finally, the authors show data (Tabs. 1-3) obtained based on Eq. (3), i.e., the standard deviation. The very low standard deviation in all tested diodes and conditions should be noted in this case. The average standard deviations obtained for all tested temperatures and currents are $4 \mathrm{mV}, 2.2 \mathrm{mV}$ and $1.9 \mathrm{mV}$ for NXP, ROHM and Onsemi, respectively. Such results mean a very high repeatability for each manufacturer.

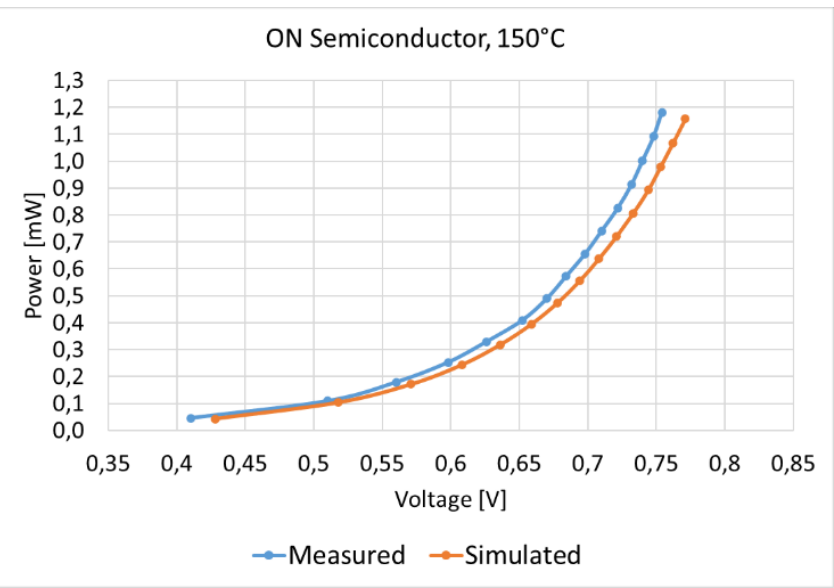

Fig. 13. Test results $(P(U))-$ Onsemi $150^{\circ} \mathrm{C}$

Tab. 1. Standard deviation - NXP

\begin{tabular}{|c|c|c|c|c|c|c|c|c|}
\hline \multicolumn{9}{|c|}{ standard deviation [V] } \\
\hline \multirow[t]{2}{*}{$\mathrm{T}\left[{ }^{\circ} \mathrm{C}\right]$} & \multicolumn{8}{|c|}{$\mathrm{I}[\mu \mathrm{A}]$} \\
\hline & 100 & 300 & 500 & 700 & 900 & 1100 & 1300 & 1500 \\
\hline-40 & 0,004 & 0,005 & 0,005 & 0,005 & 0,005 & 0,005 & 0,005 & 0,005 \\
\hline-20 & 0,003 & 0,004 & 0,005 & 0,005 & 0,006 & 0,006 & 0,006 & 0,006 \\
\hline 0 & 0,004 & 0,004 & 0,005 & 0,005 & 0,006 & 0,006 & 0,006 & 0,007 \\
\hline 25 & 0,035 & 0,009 & 0,006 & 0,006 & 0,005 & 0,005 & 0,005 & 0,007 \\
\hline 50 & 0,002 & 0,002 & 0,002 & 0,003 & 0,003 & 0,003 & 0,003 & 0,005 \\
\hline 75 & 0,002 & 0,002 & 0,002 & 0,002 & 0,002 & 0,002 & 0,002 & 0,002 \\
\hline 100 & 0,002 & 0,002 & 0,002 & 0,002 & 0,002 & 0,002 & 0,002 & 0,002 \\
\hline 125 & 0,003 & 0,003 & 0,003 & 0,003 & 0,003 & 0,003 & 0,002 & 0,002 \\
\hline 150 & 0,003 & 0,003 & 0,003 & 0,003 & 0,003 & 0,003 & 0,003 & 0,003 \\
\hline
\end{tabular}

Tab. 2. Standard deviation - Onsemi

\begin{tabular}{|c|c|c|c|c|c|c|c|c|}
\hline \multicolumn{9}{|c|}{ standard deviation [V] } \\
\hline \multirow[t]{2}{*}{$\mathrm{T}\left[{ }^{\circ} \mathrm{C}\right]$} & \multicolumn{8}{|c|}{$\mathrm{I}[\mu \mathrm{A}]$} \\
\hline & 100 & 300 & 500 & 700 & 900 & 1100 & 1300 & 1500 \\
\hline-40 & 0,001 & 0,001 & 0,001 & 0,001 & 0,001 & 0,002 & 0,002 & 0,002 \\
\hline-20 & 0,001 & 0,001 & 0,001 & 0,001 & 0,001 & 0,001 & 0,001 & 0,003 \\
\hline 0 & 0,002 & 0,001 & 0,001 & 0,001 & 0,001 & 0,001 & 0,001 & 0,001 \\
\hline 25 & 0,015 & 0,003 & 0,002 & 0,001 & 0,001 & 0,001 & 0,001 & 0,002 \\
\hline 50 & 0,002 & 0,001 & 0,001 & 0,001 & 0,001 & 0,001 & 0,001 & 0,001 \\
\hline 75 & 0,003 & 0,002 & 0,001 & 0,001 & 0,001 & 0,001 & 0,001 & 0,001 \\
\hline 100 & 0,003 & 0,003 & 0,002 & 0,002 & 0,002 & 0,002 & 0,001 & 0,001 \\
\hline 125 & 0,004 & 0,003 & 0,003 & 0,002 & 0,002 & 0,002 & 0,002 & 0,002 \\
\hline 150 & 0,005 & 0,004 & 0,004 & 0,003 & 0,003 & 0,003 & 0,003 & 0,003 \\
\hline
\end{tabular}

Fig. 14 shows a geometrical analysis of Eq. (5), which determines the surface area $(D)$, representing the sum of deviations $U$ for the entire analysed current range. The $g(x)$ function represents a real-valued function based on laboratory measurements (logarithmic function), while the $f(x)$ function is a linear-ideal function. The difference in the course of the $f(x)$ and $g(x)$ functions arises 
from the difference between an ideal plot of the $g(x)$ straight line and the $g(x)$ function, which represents the laboratory test results. The resulting surface area $(D)$ was created under the assumption that the starting and ending measurement points were common for them, which stems from the assumption and factor analysed during this test.

Tab. 3. Standard deviation - ROHM

\begin{tabular}{|c|c|c|c|c|c|c|c|c|}
\hline \multicolumn{9}{|c|}{ standard deviation [V] } \\
\hline \multirow[t]{2}{*}{$\mathrm{T}\left[{ }^{\circ} \mathrm{C}\right]$} & \multicolumn{8}{|c|}{$\mathrm{I}[\mu \mathrm{A}]$} \\
\hline & 100 & 300 & 500 & 700 & 900 & 1100 & 1300 & 1500 \\
\hline-40 & 0,001 & 0,001 & 0,001 & 0,001 & 0,001 & 0,001 & 0,001 & 0,001 \\
\hline-20 & 0,001 & 0,001 & 0,001 & 0,001 & 0,001 & 0,001 & 0,001 & 0,003 \\
\hline 0 & 0,002 & 0,002 & 0,002 & 0,002 & 0,001 & 0,002 & 0,002 & 0,003 \\
\hline 25 & 0,010 & 0,003 & 0,002 & 0,002 & 0,002 & 0,002 & 0,002 & 0,002 \\
\hline 50 & 0,003 & 0,002 & 0,002 & 0,002 & 0,002 & 0,002 & 0,002 & 0,003 \\
\hline 75 & 0,003 & 0,003 & 0,003 & 0,002 & 0,002 & 0,002 & 0,002 & 0,002 \\
\hline 100 & 0,003 & 0,003 & 0,003 & 0,003 & 0,003 & 0,003 & 0,003 & 0,003 \\
\hline 125 & 0,003 & 0,003 & 0,003 & 0,003 & 0,003 & 0,003 & 0,003 & 0,003 \\
\hline 150 & 0,003 & 0,003 & 0,003 & 0,003 & 0,003 & 0,003 & 0,003 & 0,004 \\
\hline
\end{tabular}

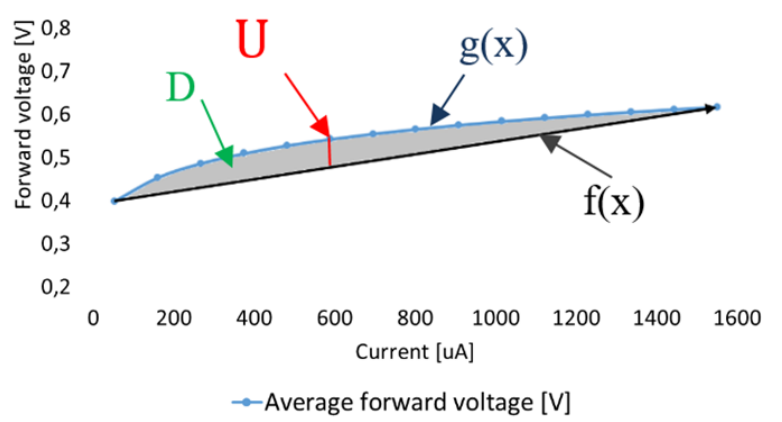

Fig. 14. Determination of the linearity coefficient $(D)$

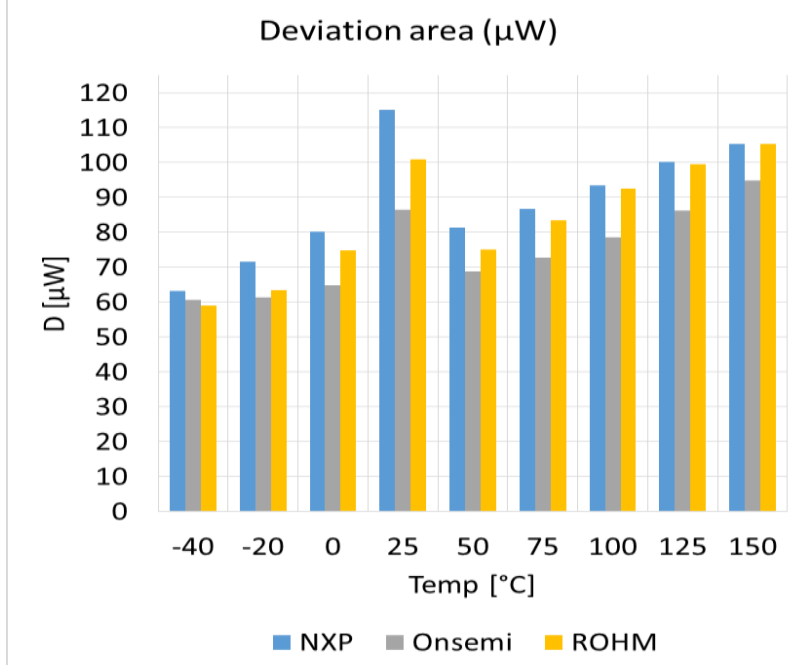

Fig. 15. Deviation area $(D)$

Fig. 15 shows the results obtained for each manufacturer, based on Eq. (3), for each of the nine analysed ambient temperatures - (blue - NXP, grey - Onsemi, yellow - ROHM). The higher the deviation area value, the higher the deviation from the linear, ideal course of $f(x)$ shown in Fig. 14

Finally, the results of measurements were compared with datasheets. It was calculated that $98.9 \%$ of the tested elements were in the range of \pm six sigma. Further calculations were made to better assess the phenomenon. The significance of the linear one-dimensional regression function was tested. The previously calculated means and standard deviations were used, and then the moment of correlation of the variables from the sample was calculated. Successively, the linear correlation coefficient was obtained, which characterizes the degree of linear dependence between random variables. For the analysed case, the correlation coefficient is 0.95 and the $t$ factor for the analysed case is 67.94 . Based on the results obtained for the moment of linear correlation and its coefficient, it was concluded that there is a very close relationship between the analysed data and the hypothesis that there is no correlation between the analysed variables should be rejected.

More about this analysis can be found [32, 33].

\section{CONCLUSIONS}

The article describes a methodology for analysing electronic circuits based on a module acting as a resistor calibrator, which can also work as a temperature sensor. The WCA, which is the key verifier of a correctly designed system, was expanded in this study with a deeper analysis, based on large-scale laboratory tests. The analysis of standard deviations, maximum deviations from the mean and obtained deviation areas for individual temperatures and manufacturers provided a much better picture of a component's behaviour under expected operating conditions, compared with manufacturer's documentation. It should be noted that the standard deviations for the measurement of $25^{\circ} \mathrm{C}$ and $100 \mu \mathrm{A}$ differ significantly from the rest of the results obtained. The reason for such a situation is the human factor that has serially influenced the measurements at this particular temperature and in this particular flowing current. These measurements should be disregarded when drawing conclusions for the entire study. The obtained results and comparisons for the rest of the measured and simulated data prove that such an approach is justified.

Based on the standard deviations, it can be concluded that the NXP diode slightly differs from the other two manufacturers, due to the complete current and temperature range. When analysing the deviation area, it can be noticed that Onsemi diodes exhibit lower deviations for almost all of the tested ambient temperatures than the semiconductor of other manufacturers. The average deviation area offset for all temperatures and current levels is $89 \mu \mathrm{W}, 84 \mu \mathrm{W}$ and $75 \mu \mathrm{W}$ for NXP, ROHM and Onsemi, respectively.

All analysed diodes are characterized by very similar calculation results. It is difficult to unequivocally select the best manufacturer. However, ultimately, based on the conducted calculations, Onsemi diodes should be considered the best product. They exhibit the most linear $I_{F}\left(V_{F}\right)$ operating range and the highest result repeatability, which is demonstrated by the results of standard deviation calculations.

\section{REFERENCES}

1. Smith W. M. Worst case circuit analysis-an overview (electronic parts/circuits tolerance analysis), Proc. Annu. Reliab. Maintainab. Symp. 1996; 326-334. 
2. Anceaume A., Cabillic G., Chevochot P., Puaut I. A middleware support for distributed safety-critical real-time applications, In Proc. of the 18th Inter. Conf. Distrib. Comp. Syst. 1998; 344-351.

3. Colin A., Bernat G. Scope-tree: A program representation for symbolic worst-case execution time analysis, Proc. 14th Euromicro Conf. of Real-Time Syst. 2002; 50-59.

4. Colin A., Puaut I. Worst case execution time analysis for a processor with branch prediction, Real-Time Syst. 2000;18: 249-274.

5. Carlsson M., Engblom J., Ermedahl A., Lindblad J., Lisper B. Worstcase execution time analysis of disable interrupt regions in a commercial real-time operating system, Proc. Inter. Work. Real-Time Tools. 2002; 1-12.

6. Engblom J., Static properties of embedded real-time programs and their implications for worst-case execution time analysis, Proc. IEEE Real-Time Tech. Appl. Symp. 1999; 46-55.

7. Jacques S., Batut N., Leroy R., Gonthier L. Aging test results for high temperature triacs during power cycling, Proc. IEEE Pwr. Electro. Spec. Conf. 2008; 2447-2452.

8. Lien W., Damrongplasit N., Paredes J.H., Senesky D.G., Liu T.K., Pisano A.P. 4H-SiC N-Channel JFET for operation in hightemperature environments, IEEE J. Electron Devices Soc. 2014; 2(6): 4-7

9. Shwarts Y. M., Sokolov V. N., Shwarts M. M., Fedorov I. A., Venger E. F. Advanced silicon diode temperature sensors with minimized self-heating and noise for cryogenic applications, Proc. Inter. Euro. Conf. Adv. Semicond. Dev. Microsyst. 2000; 351-354.

10. Ferlet-Cavrois V., Colladant T., Paillet P., Leray J.L., Musseau O., Worst-case bias during total dose irradiation of SOI transistors, IEEE Trans. Nuc. Sci. 2000; 47(6): 2183 - 2188.

11. Lynch W. T. Wosrt-Case Analysis of a fesistor memory matrix, IEEE Trans. Comput. 1969; 18(10): 940-942.

12. Marcovitz M., Seif E. Analytical design of resistor-coupled transistor logical circuit, IRE Trans. Elect. Comp. 1958; 7(2): 109-119.

13. Tian W., Ling X. T., Liu R.W. Novel methods for circuit worst-case tolerance analysis, IEEE Trans. Circuits Syst. I. Fundam. Theory Appl. 1996; 43(4): 272-278.

14. Donnelly T.J., Pekarek S.D., Fudge D., Vaks N., Zarate N. Predicting Worst Case Common-Mode behavior in power electronic based systems, IEEE Electr. Ship. Tech. Symp. 2019; 396-402.

15. Spence R., Soin R. S., Tolerance design of electronic circuits, Imperial College Press; 1988.

16. Divekar D.A. DC statistical circuit analysis for bipolar IC's using parameter correlations - an experimental example, leee T Comput Aid D. 2006; 101-103.

17. Riley J. C. The accuracy of series and parallel connections of fourteminal resistors, IEEE Trans. Instrum. Meas. 1967; 16(3): 258-268.
18. Sokół K., Ptak P. Experimental verification of mathematical models for failure estimation of electronic systems, Acta Phys. Pol. A 2020; 2(138): 207-209.

19. Hillebrand M., Paul T. Dealing with $1 / O$ devices in the context of pervasive system verification, Inter. Conf. Comp. Dsg. 2005; 309316.

20. Nassif S.R., Strojwas A.J., Director S.W. A methodology for WorstCase Analysis of integrated circuits, leee leee T Comput Aid D. 1986; 5(1): $104-113$.

21. Rafaila M., Decker C., Grimm C., Pelz G. Simulation-based sensitivity and worst-case analyses of automotive electronics, IEEE Symp. Dsg. Diag. Elec. Circ. Syst., 2010; 309-312.

22. Maly W., Strojwas A.J. Statistical simulation of the IC manufacturing process, IEEE Trans. Comput.-Aided Design Integr. Circuits Syst. 1982; 1(3): 120-131.

23. Nassif S.R., Strojwas A.J., Director S.W. FABRICS II: A statistically based IC fabrication process simulator, IEEE Trans. Comput.-Aided Design Integr. Circuits Syst. 1984; 3(1): 40-46,

24. Lanchester P. C. Digital thermometer circuit for silicon diode sensors, Cryogenics 1989; 29 (12): 1156-1159.

25. Mansoor M., Haneef I., Akhtar S., De Luca A., Udrea F. Silicon diode temperature sensors $-A$ review of applications, Sens. Actuator $A$ Phys. 2015; 232 (1): 63-74.

26. Szmyrka-Grzebyk A., Lipiński L. Linear diode thermometer in the 4300 K temperature range, Cryogenics 1995; 35(4): 281-284.

27. Efron B., Tibshirani R. Statistical data analysis in the computer age, Science 1991; 253 (5018): 390-395.

28. Zięba A. Analiza danych w naukach ścisłych i technice [Data analysis in exact sciences and technology], Wydawnictwo Naukowe PWN; 2013.

29. Harry M., Ronald Lawson J. Six Sigma Producibility Analysis and Process Characterization, Addison-Wesley; 1992.

30. Yang K., El-Haik B. Design for six sigma: A Roadmap for Product Development, The MacGraw-Hill Companies; 2003.

31. White R. V. An Introduction to Six Sigma with a design example, Ann. Appl. Pwr. Elec. Conf. Exps. 1992; 28-35.

32. Majchrzak E., Mochnacki B. Metody numeryczne [Numerical methods], Wydawnictwo Politechniki Śląskiej; 2004.

33. Sorokin A. E. Experimental verification of a mathematical model for a heat store, Russ. Eng. Res. 2021; 41: 742-744.

Krzysztof Sokół: (ID https://orcid.org/0000-0002-8661-1763

Piotr Ptak: iD https://orcid.org/0000-0002-2477-0136 